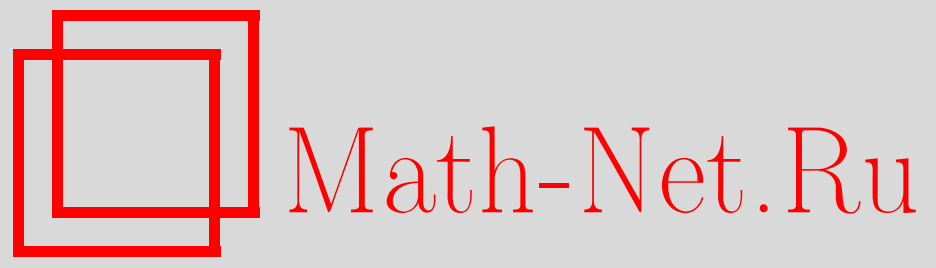

J. Kallsen, $\sigma$-localization and $\sigma$-martingales, Теория вероятн. и ее примен., 2003, том 48, выпуск 1, 177-188

DOI: https://doi.org/10.4213/tvp309

Использование Общероссийского математического портала Math-Net.Ru подразумевает, что вы прочитали и согласны с пользовательским соглашением

http://www . mathnet.ru/rus/agreement

Параметры загрузки:

IP : 3.85 .73 .92

26 апреля 2023 г., 10:53:56

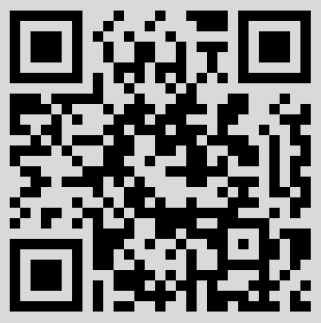




\title{
REFERENCES
}

1. Christopeit $N$. The pricing of perpetual American options in a binomial model. Discussion paper. Bonn: Institute for Econometrics, 2001.

2. Neveu J. Discrete-Parameter Martingales. Amsterdam: North-Holland, 1975, 236 p.

3. Ширяев А.Н. Статистический последовательный анализ. Оптимальные правила остановки. М.: Наука, 1976, 272 с.

4. ШШияев А.Н. Основы стохастической финансовой математики. Т. 1: Факты, модели. Т. 2: Теория. М.: ФАЗИС, 1998, 512 с., 544 с.

5. Ширяев А. Н., Кабанов Ю. М., Крамков Д. О., Мельников А. В. К теории расчетов опционов Европейского и Американского типов. І. Дискретное время. - Теория вероятн. и ее примен., 1994, т. 39, в. 1, с. 23-79.

Поступила в редакцию

27.III. 2002

(c) 2003 r.

KALLSEN J.*

\section{$\sigma$-LOCALIZATION AND $\sigma$-MARTINGALES}

\begin{abstract}
В статье вводится понятие $\sigma$-локализации, обобщающее понятие локализации в общей теории случайных процессов. $\sigma$-локализационный класс, связанный с множеством мартингалов, есть класс $\sigma$-мартингалов, который играет важную роль в финансовой математике. Подробно рассматриваются эти процессы и соответствующие $\sigma$-мартингальные меры. Обобщая понятие стохастического интеграла по компенсированным случайным мерам, мы выводим каноническое представление для $\sigma$-мартингалов.
\end{abstract}

Ключевые слова и фразы: $\sigma$-локализация, $\sigma$-мартингал, стохастический интеграл, каноническое представление, $\sigma$-мартингальная мера.

1. Introduction. $\sigma$-Martingales have been introduced by Chou [3] and were investigated further by Emery [6]. They play a key role in the general statement of the fundamental theorems of asset pricing in [5], [12], and [2]. $\sigma$-Martingales can be interpreted quite naturally as semimartingales with vanishing drift. Similar to local martingales, the set of $\sigma$-martingales may be obtained from the class of martingales by a localization procedure, but here localization has to be understood in a broader sense than usually (cf. $[4, \mathrm{I} .1 \mathrm{~d}]$ ). This concept of $\sigma$-localization is introduced in Section 2 . The subsequent section treats the set of $\sigma$-martingales and their properties. By extending the stochastic integral relative to compensated random measures, the canonical local martingale representation $X=X_{0}+X^{c}+x *(\mu-\nu)$ is generalized to $\sigma$-martingales in Section 4 . Finally, $\sigma$-martingale measures are characterized in terms of semimartingale characteristics.

Throughout the paper, we use the notation of [4] and [9], [10]. In particular, we work with a filtered probability space $\left(\Omega, \mathscr{F},\left(\mathscr{F}_{t}\right)_{t \in \mathbf{R}_{+}}, P\right)$. The transposed of a vector $x$ or matrix is denoted by $x^{\top}$ and its components by superscripts. Increasing processes are identified with their corresponding Lebesgue-Stieltjes measure.

* Institut für Mathematische Stochastik, Universität Freiburg, Eckerstraße 1, D-79104 Freiburg i. Br., Germany; e-mail: kallsen@stochastik.uni-freiburg.de 
2. $\sigma$-Localization. For any semimartingale $X$ and any predictable set $D \subset \Omega \times \mathbf{R}_{+}$, we write $X^{D}:=X_{0} 1_{D}(0)+1_{D} \cdot X$, where $1_{D}(0)(\omega):=1_{D}((\omega, 0))$ for $\omega \in \Omega$. In particular, we have $X^{\llbracket 0, T \rrbracket}=X^{T}$ for any stopping time $T$ (cf. [4, I.4.37]).

$\mathrm{D}$ e f i n it i o n 2.1. For any class $\mathscr{C}$ of semimartingales we define the $\sigma$-localized class $\mathscr{C}_{\sigma}$ as follows: A process $X$ belongs to $\mathscr{C}_{\sigma}$ if and only if there exists an increasing sequence $\left(D_{n}\right)_{n \in \mathbf{N}}$ of predictable sets such that $D_{n} \uparrow \Omega \times \mathbf{R}_{+}$up to an evanescent set and $X^{D_{n}} \in \mathscr{C}$ for any $n \in \mathbf{N}$.

$\mathrm{D}$ e $\mathrm{f}$ i $\mathrm{n}$ i t i o $\mathrm{n} 2.2$. A class $\mathscr{C}$ of semimartingales is called stable under $\sigma$-stopping if $X^{D} \in \mathscr{C}$ for any $X \in \mathscr{C}$ and any predictable set $D$.

Lemma 2.1. If $\mathscr{C}$ is stable under stopping, then $\left(\mathscr{C}_{\text {loc }}\right)_{\sigma}=\mathscr{C}_{\sigma}$.

$\mathrm{P}$ r o of. We mimic the proof of a similar statement in [4, I.1.35]. Let $X \in\left(\mathscr{C}_{\text {loc }}\right)_{\sigma}$ and $\left(D_{n}\right)_{n \in \mathbf{N}}$ a localizing sequence of predictable sets such that $X^{D_{n}} \in \mathscr{C}_{\text {loc }}$ for any $n \in \mathbf{N}$. Since $\mathscr{C}_{\text {loc }}$ is stable under stopping, we may assume that $D_{n}=D_{n} \cap \llbracket 0, n \rrbracket$. For any $n \in \mathbf{N}$ there exist a localizing sequence of stopping times $(T(n, p))_{p \in \mathbf{N}}$ and $p_{n} \in \mathbf{N}$ such that $\left(X^{D_{n}}\right)^{T(n, p)} \in \mathscr{C}$ for any $p \in \mathbf{N}$ and $P\left(T\left(n, p_{n}\right)<n\right) \leqslant 2^{-n}$. Let $\widetilde{D}_{n}:=D_{n} \cap\left(\bigcap_{m \geqslant n} \llbracket 0, T\left(m, p_{m}\right) \rrbracket\right)$. Observe that $\left(\widetilde{D}_{n}\right)_{n \in \mathbf{N}}$ is an increasing sequence of predictable sets. Let $k \in \mathbf{N}$ and $(\omega, t) \in \Omega \times[0, k]$ with $(\omega, t) \in\left(\bigcup_{n \in \mathbf{N}} D_{n}\right) \backslash\left(\bigcup_{n \in \mathbf{N}} \widetilde{D}_{n}\right)=$ $\lim \sup _{n \rightarrow \infty}\left(D_{n} \backslash \widetilde{D}_{n}\right)$. Obviously, this holds (up to evanescence) also for $(\omega, k)$ instead of $(\omega, t)$. Since

$$
\begin{aligned}
& P\left(\left\{\omega \in \Omega:(\omega, k) \in D_{n} \backslash \widetilde{D}_{n}\right\}\right) \leqslant \sum_{m \geqslant n} P\left(T\left(m, p_{m}\right)<n\right) \\
& \quad \leqslant \sum_{m \geqslant n} P\left(T\left(m, p_{m}\right)<m\right) \leqslant \sum_{m \geqslant n} 2^{-m}=2^{-(n-1)},
\end{aligned}
$$

the Borel-Cantelli lemma yields $P\left(\left\{\omega \in \Omega:(\omega, k) \in \limsup _{n \rightarrow \infty}\left(D_{n} \backslash \widetilde{D}_{n}\right)\right\}\right)=0$ and hence $\Omega \times[0, k] \subset \bigcup_{n \in \mathbf{N}} \widetilde{D}_{n}$ up to an evanescent set. Since $\mathscr{C}$ is stable under stopping

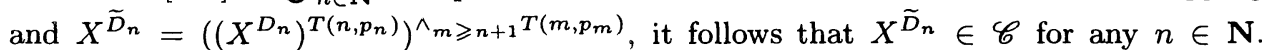
Hence $X \in \mathscr{C}_{\sigma}$. Lemma 2.1 is proved.

The following result serves primarily as a preparation to Corollary 2.1 because most classes of interest (in particular $\mathscr{M}$ ) are not stable under $\sigma$-stopping.

Proposition 2.1. If $\mathscr{C}$ is stable under $\sigma$-stopping, then $\left(\mathscr{C}_{\sigma}\right)_{\sigma}=\mathscr{C}_{\sigma}$.

$\mathrm{P}$ r o of. The proof is similar to the proof of Lemma 2.1. Let $X \in\left(\mathscr{C}_{\sigma}\right)_{\sigma}$ and $\left(D_{n}\right)_{n \in \mathbf{N}}$ a localizing sequence of predictable sets such that $X^{D_{n}} \in \mathscr{C}_{\sigma}$ for any $n \in \mathbf{N}$. Let the characteristics of $X$ be given in the form (3.1) below. Without loss of generality we may modify $A$ so that $A_{0-}=0, \Delta A_{0}=A_{0}-A_{0_{-}}>0$, and $A_{\infty}=1$, which implies that $P \otimes A$ is a probability measure on $\left(\Omega \times \mathbf{R}_{+}, \mathscr{P}\right)$. For any $n \in \mathbf{N}$ there exist a localizing sequence of predictable sets $(D(n, p))_{p \in \mathbf{N}}$ and $p_{n} \in \mathbf{N}$ such that $\left(X^{D_{n}}\right)^{D(n, p)} \in \mathscr{C}$ for any $p \in \mathbf{N}$ and $(P \otimes A)\left(D\left(n, p_{n}\right)^{C}\right) \leqslant 2^{-n}$. Let $\widetilde{D}_{n}:=D_{n} \cap\left(\bigcap_{m \geqslant n} D\left(m, p_{m}\right)\right)$. Observe that $\left(\widetilde{D}_{n}\right)_{n \in \mathbf{N}}$ is an increasing sequence of predictable sets and hence $\left(\bigcup_{n \in \mathbf{N}} D_{n}\right) \backslash\left(\bigcup_{n \in \mathbf{N}} \widetilde{D}_{n}\right)=\lim \sup _{n \rightarrow \infty}\left(D_{n} \backslash \widetilde{D}_{n}\right)$. Since $(P \otimes$ $A)\left(D_{n} \backslash \widetilde{D}_{n}\right) \leqslant \sum_{m \geqslant n}(P \otimes A)\left(D\left(m, p_{m}\right)^{C}\right) \leqslant \sum_{m \geqslant n} 2^{-m}=2^{-(n-1)}$, the Borel-Cantelli lemma yields $(P \otimes A)\left(\limsup \operatorname{sum}_{n \rightarrow \infty}\left(D_{n} \backslash \widetilde{D}_{n}\right)\right)=0$. Therefore, $D:=\left(\Omega \times \mathbf{R}_{+}\right) \backslash\left(\bigcup_{n \in \mathbf{N}} \widetilde{D}_{n}\right)$ is a $(P \otimes A)$-null set. By [14, Lemma 2.5], this implies that $X_{0} 1_{D}(0)+1_{D} \cdot X=0$ and hence $X^{\widetilde{D}_{n} \cup D}=X^{\widetilde{D}_{n}}$ up to indistinguishability. Since $\mathscr{C}$ is stable under $\sigma$-stopping and $X^{\widetilde{D}_{n} \cup D}=X^{\widetilde{D}_{n}}=\left(\left(X^{D_{n}}\right)^{D\left(n, p_{n}\right)}\right)^{n_{m} \geqslant n+1}{ }^{D\left(m, p_{m}\right)}$, it follows that $X^{\widetilde{D}_{n} \cup D} \in \mathscr{C}$ for any $n \in \mathbf{N}$. Hence $X \in \mathscr{C}_{\sigma}$. Proposition 2.1 is proved.

Corollary 2.1. If $\mathscr{C}$ is stable under stopping and $\mathscr{C}_{\text {loc }}$ is stable under $\sigma$-stopping, then $\mathscr{C}_{\sigma}$ is stable under $\sigma$-stopping and $\left(\mathscr{C}_{\sigma}\right)_{\sigma}=\mathscr{C}_{\sigma}$.

$\mathrm{P}$ r o of. It is easy to see that $\left(\mathscr{C}_{\text {loc }}\right)_{\sigma}$ is stable under $\sigma$-stopping, which implies that $\mathscr{C}_{\sigma}$ is stable under $\sigma$-stopping (cf. Lemma 2.1). By Lemma 2.1 and Proposition 2.1, we have $\left(\mathscr{C}_{\sigma}\right)_{\sigma}=\left(\left(\mathscr{C}_{\text {loc }}\right)_{\mathcal{C}}\right)_{\sigma}=\left(\mathscr{C}_{\text {loc }}\right)_{\sigma}=\mathscr{C}_{\sigma}$. 
The following result shows that the general stochastic integral in [10] can easily be interpreted in terms of $\sigma$-localization.

Lemma 2.2. Let $X$ be an $\mathbf{R}^{d}$-valued semimartingale and $H$ an $\mathbf{R}^{d}$-valued predictable process. Then $H \in L(X)$ if and only if there exist a semimartingale $Z$ and an increasing sequence $\left(D_{n}\right)_{n \in \mathbf{N}}$ of predictable sets such that $D_{n} \uparrow \Omega \times \mathbf{R}_{+}$up to an evanescent set, $H 1_{D_{n}}$ is bounded, and $Z^{D_{n}}=\left(H 1_{D_{n}}\right)^{\top} \cdot X$ for any $n \in \mathbf{N}$. In this case $Z=H^{\top} \cdot X$.

P r o of. $\Rightarrow$ : Let $D_{n}:=\{|H| \leqslant n\}$ for any $n \in \mathbf{N}$. The statement follows from $[7$, Proposition A.1].

$\Leftarrow$ : On any $D_{n}$ we have $\Delta Z=\Delta Z^{D_{n}}=\left(H 1_{D_{n}}\right)^{\top} \Delta X=H^{\top} \Delta X$, which implies that $D:=\{|\Delta X|>1\} \cup\left\{\left|H^{\top} \Delta X\right|>1\right\}$ is a discrete set in the sense of [10]. Let $M$ be the local martingale part of the $\mathbf{R}^{d}$-valued special semimartingale $\bar{X}:=$ $X-X_{0}-\sum_{t \leqslant} \Delta X_{t} 1_{D}(t)$ and $A:=X-M$, which has components in $\mathscr{V}$. Choose an $\mathbf{R}^{d \times d}$-valued process $c$ and $C \in \mathscr{V}$ such that $\left[M^{i}, M^{j}\right]=c^{i j} \cdot C$ for $i, j=1, \ldots, d$. Moreover, choose an $\mathbf{R}^{d}$-valued optional process $a$ and $\bar{A} \in \mathscr{V}$ with $A^{i}=a^{i} \cdot \bar{A}$ for $i=1, \ldots, d$. Finally, let $N$ be the local martingale part of the special semimartingale $\bar{Z}:=Z-\sum_{t \leqslant \cdot} \Delta Z_{t} 1_{D}(t)$ and $B:=Z-N \in \mathscr{V}$. Since $\bar{Z}$ has bounded jumps, we have $N \in \mathscr{H}_{\text {loc }}^{2}$ (cf. [4, I.4.24]). Fix $n \in$ N. Since $H 1_{D_{n}}$ is locally bounded and $\left(H 1_{D_{n}}\right)^{\top} \cdot \bar{X}=$ $\left(H 1_{D_{n}}\right)^{\top} \cdot X-\sum_{t \leqslant .}\left(H_{t} 1_{D_{n}}(t)\right)^{\top} \Delta X_{t} 1_{D}(t)=Z^{D_{n}}-\sum_{t \leqslant \cdot} \Delta Z_{t}^{D_{n}} 1_{D}(t)=\bar{Z}^{D_{n}}$, Proposition 2 in [10] yields that $\left(H 1_{D_{n}}\right)^{\top} \cdot M=N^{D_{n}}$ and hence $\left(H 1_{D_{n}}\right)^{\top} \cdot A=B^{D_{n}}$. Therefore $1_{D_{n}} \cdot[N, N]=\left[N^{D_{n}}, N^{D_{n}}\right]=\left[\left(H 1_{D_{n}}\right)^{\top} \cdot M,\left(H 1_{D_{n}}\right)^{\top} \cdot M\right]=\left(H^{\top} c H 1_{D_{n}}\right) \cdot C$, which implies that $\left(H^{\top} c H\right) \cdot C=[N, N] \in \mathscr{A}_{\text {loc }}^{+}$by monotone convergence. In particular, $H \in L_{\text {loc }}^{2}(M) \subset$ $L_{\text {loc }}^{1}(M)$. Moreover, $1_{D_{n}} \cdot \operatorname{Var}(B)=\operatorname{Var}\left(B^{D_{n}}\right)=\left|\sum_{i=1}^{d}\left(H^{i} 1_{D_{n}} a^{i}\right)\right| \cdot \bar{A}$, where $\operatorname{Var}(B)$ denotes the variation process of $B \in \mathscr{V}$. Again by monotone convergence, this implies $\left|\sum_{i=1}^{d}\left(H^{i} a^{i}\right)\right| \cdot \bar{A}=\operatorname{Var}(B) \in \mathscr{V}$ and hence $H \in L_{s}(A)$. Altogether, we have $H \in L(X)$. The equality $Z=H^{\top} \cdot X$ follows from the first part of the proof. Lemma 2.2 is proved.

3. $\sigma$-Martingales. Of course, we have $\mathscr{C} \subset \mathscr{C}_{\text {loc }} \subset \mathscr{C}_{\sigma}$ for any class $\mathscr{C}$ of semimartingales. As a particular case, we obtain the set $\mathscr{M}_{\sigma}$ of $\sigma$-martingales. Denote by $\mathscr{D}$ the set of semimartingales such that the stopped process $X^{t}$ is of class (D) for any $t \in \mathbf{R}_{+}$ (cf. $\left[4\right.$, I.1.46]). Below we will consider the corresponding localized classes $\mathscr{D}_{\text {loc }}$ and $\mathscr{D}_{\sigma}$.

The name $\sigma$-martingale was used in [5] to refer to the set of semimartingales de la classe $\left(\Sigma_{m}\right)$ introduced by Chou [3] and Emery [6], who also consider $\mathscr{D}_{\sigma}$ as semimartingales de la classe $(\Sigma)$. To be more precise, $X \in \mathscr{M}_{\sigma}$ (respectively, $X \in \mathscr{D}_{\sigma}$ ) holds if and only if $X-X_{0}$ is a semimartingale de la classe $\left(\Sigma_{m}\right)$ (respectively, semimartingale de la classe $(\Sigma)$ ). Some researchers prefer the older name martingale transform which was originally applied in discrete-time settings. Equivalent definitions can be found in $[6$, Proposition 2].

The $\sigma$-martingale property can easily be read from the characteristics of a semimartingale as it was observed by Kabanov [12, Lemma 3]. To this end, fix a real-valued semimartingale $X$ with $X_{0}=0$. Let its characteristics $(B, C, \nu)$ relative to some truncation function $h: \mathbf{R} \rightarrow \mathbf{R}$ be given in the form

$$
B=b \cdot A, \quad C=c \cdot A, \quad \nu=A \otimes F,
$$

where $A \in \mathscr{A}_{\text {loc }}^{+}$and $b$ are predictable processes, $c$ is a predictable $\mathbf{R}_{+}$-valued process, and $F$ is a transition kernel from $\left(\Omega \times \mathbf{R}_{+}, \mathscr{P}\right)$ into $(\mathbf{R}, \mathscr{B})$ (cf. $[4$, II.2.9]).

Lemma 3.1. 1. $X$ is a $\sigma$-martingale if and only if $\int_{\{|x|>1\}}|x| F(d x)<\infty$ and

$$
b+\int(x-h(x)) F(d x)=0
$$

hold $(P \otimes A)$-almost everywhere on $\Omega \times \mathbf{R}_{+}$.

2. $X$ is a local martingale if and only if $\int_{\{|x|>1\}}|x| F(d x) \in L(A)$ and equation (3.2) holds $(P \otimes A)$-almost everywhere on $\Omega \times \mathbf{R}_{+}$.

3. $X$ is a martingale if and only if $X \in \mathscr{D}$ and equation (3.2) holds $(P \otimes A)$-almost everywhere on $\Omega \times \mathbf{R}_{+}$. 
$\mathrm{P} \mathrm{r}$ o o f. 2. This follows immediately from [4, II.2.29, II.2.13, I.3.10].

1. This follows from Statement 2 because $\mathscr{M}_{\sigma}=\left(\mathscr{M}_{\text {loc }}\right)_{\sigma}$ by Lemma 2.1 (cf. also [14, Lemma 2.5]).

3. $\Rightarrow$ : Since martingales are local martingales that belong to $\mathscr{D}$ (cf. $[4, \mathrm{I} .1 .47])$, this inclusion follows from Statement 2.

$\Leftarrow$ : By [9, Exercise 2.1], we have that $\mathscr{D}_{\text {loc }}$ coincides with the set of special semimartingales, which in turn satisfy $\int_{\{|x|>1\}}|x| F(d x) \in L(A)$ (cf. [4, II.2.29]). Using Statement 2 we conclude that $X$ is a local martingale of class (D) on $[0, t]$ for any $t \in \mathbf{R}_{+}$and hence a martingale (cf. [4, I.1.47]). Lemma 3.1 is proved.

Consequently, martingales, local martingales, and $\sigma$-martingales can all be interpreted as processes with vanishing drift (3.2) that differ only in the extent of uniform integrability. If we drop equation (3.2) and keep the integrability conditions in Lemma 3.1, then we end up with the sets $\mathscr{D}, \mathscr{D}_{\text {loc }}, \mathscr{D}_{\sigma}$ as the following lemma shows.

Lemma 3.2. 1. $X \in \mathscr{D}_{\sigma}$ if and only if $\int_{\{|x|>1\}}|x| F(d x)<\infty(P \otimes A)$-almost everywhere on $\Omega \times \mathbf{R}_{+}$.

2. $X \in \mathscr{D}_{\text {loc }}$ if and only if $X$ is a special semimartingale if and only if $\int_{\{|x|>1\}}|x| F(d x) \in L(A)$.

$\mathrm{P} \mathrm{r}$ o o f. 2. The first equivalence is [9, Exercise 2.1]. The second equivalence follows from $[4$, II.2.29, II.2.13, I.3.10].

1. Since $\mathscr{D}_{\sigma}=\left(\mathscr{D}_{\text {loc }}\right)_{\sigma}$ by Lemma 2.1, this follows from Statement 2.

Let us summarize some relationships between $\sigma$-martingales and other classes of processes.

Corollary 3.1. 1. $X$ is a local martingale if and only if it is both a $\sigma$-martingale and a special semimartingale.

2. $X$ is a martingale if and only if it is both a $\sigma$-martingale and in $\mathscr{D}$.

3. $X$ is a uniformly integrable martingale if and only if it is a $\sigma$-martingale of class (D).

$\mathrm{P} \mathrm{r}$ o o f. This follows immediately from Lemmas 3.1, 3.2 and [4, I.1.47].

Corollary 3.2. 1. Any locally bounded $\sigma$-martingale is a local martingale.

2. Any bounded $\sigma$-martingale is a uniformly integrable martingale.

$\mathrm{P}$ r o o f. Corollary $3.1,[4$, I.4.23].

Note that any discrete-time $\sigma$-martingale starting in 0 (i.e., of the form $X=$ $\left.\sum_{t \leqslant} . \Delta X_{t} 1_{\mathbf{N} \backslash\{0\}}(t)\right)$ is a local martingale because the integrability conditions in Statements 1 and 2 of Lemma 3.2 are equivalent for $A=\sum_{t \leqslant .} .1_{N \backslash\{0\}}(t)$.

The following result generalizes a parallel statement for local supermartingales (cf. $[9,(5.17)])$. It is needed, e.g., in [8].

Proposition 3.1. Let $X$ be a nonnegative $\sigma$-supermartingale with $E\left(X_{\mathrm{C}}\right)<\infty$. Then $X$ is a supermartingale.

$\mathrm{P} \mathrm{r}$ o o f. Let the characteristics of $X$ be of the form (3.1). Since any supermartingale is locally of class (D) (cf. [9, Exercise 2] or the proof of $[9,(2.18)]$ ), there exists a sequence $\left(D_{n}\right)_{n \in \mathbf{N}}$ of predictable sets with $D_{n} \uparrow \Omega \times \mathbf{R}_{+}$such that $1_{D_{n}} \cdot X$ is a supermartingale of class (D) for any $n$.

Fix $n \in \mathbf{N}$. By $\left[4\right.$, IX.5.3] or [14, Lemma 2.5], the characteristics of $1_{D_{n}} \cdot X$ are of the form (3.1) as well, but with $b^{\prime}:=1_{D_{n}} b, c^{\prime}:=1_{D_{n}} c, F^{\prime}:=1_{D_{n}} F$ instead of $b, c, F$. Since $1_{D_{n}} \cdot X$ is a supermartingale, it is a special semimartingale and its unique predictable part of bounded variation is given by $\left(b^{\prime}+\int(x-h(x)) F^{\prime}(d x)\right) \cdot A=$ $\left(\left(b+\int(x-h(x)) F(d x)\right) 1_{D_{n}}\right) \cdot A$ (cf. [4, II.2.29]). This process is actually decreasing because $1_{D_{n}} \cdot X$ is a supermartingale of class (D) (cf. [4, I.3.38]). This implies that $b+\int(x-h(x)) F(d x) \leqslant 0(P \otimes A)$-almost everywhere on $D_{n}$ and hence on $\Omega \times \mathbf{R}_{+}$ because $n$ was arbitrarily chosen.

The nonnegativity of $X$ implies that $\int_{\{|x|>1\}}(-x \vee 0) F(d x) \leqslant X_{-} \int_{\{|x|>1\}} F(d x)$. By $\left[4\right.$, II.2.13] this implies $\int_{\{|x|>1\}}(-x \vee 0) F(d x) \in L(A)$. Since $b+\int(x-h(x)) F(d x) \leqslant 0$, it follows that $\int_{\{|x|>1\}}|x| F(d x) \in L(A)$. By Lemma 3.2 and [4, II.2.29], this means that $X$ is a special semimartingale whose predictable part of bounded variation $\left(b+\int(x-\right.$ 
$h(x)) F(d x)) \cdot A$ is decreasing. Hence, $X$ is in fact a nonnegative local supermartingale. In view of $[9,(5.17)]$, we are done.

As opposed to local martingales and special semimartingales, the corresponding $\sigma$ localized classes are stable relative to stochastic integration:

Lemma 3.3. Let $X$ be an $\mathbf{R}^{d}$-valued semimartingale and $H \in L(X)$. Then the following statements hold:

1. If $X^{i} \in \mathscr{M}_{\sigma}$ for $i=1, \ldots, d$, then $H^{\top} \cdot X \in \mathscr{M}_{\sigma}$;

2. If $X^{i} \in \mathscr{D}_{\sigma}$ for $i=1, \ldots, d$, then $H^{\top} \cdot X \in \mathscr{D}_{\sigma}$.

$\mathrm{P} \mathrm{r}$ o o f. This follows from Lemmas 3.1, 3.2 and [14, Lemma 2.5].

So far, we have concentrated on real-valued processes. As for local martingales, it makes sense to define $\mathbf{R}^{d}$-valued $\sigma$-martingales as semimartingales whose components are $\sigma$-martingales. Following [5] one may also call an $\mathbf{R}^{d}$-valued process $X \sigma$-martingale if there exist an $\mathbf{R}^{d}$-valued martingale $M$ and a predictable, nonnegative process $\varphi$ such that $\varphi \in L\left(M^{i}\right)$ and $X^{i}=X_{0}^{i}+\varphi \cdot M^{i}$ for $i=1, \ldots, d$. By [2, Theorem 5.6] and [6, Proposition 2], the two definitions are consistent.

The existence of Doob-Meyer decompositions is a delicate issue. Whereas any $X \in \mathscr{D}$ (respectively, $X \in \mathscr{D}_{\text {loc }}$ ) can be written as a sum $X=M+A$ of a martingale (respectively, local martingale) $M$ and a predictable process $A \in \mathscr{V}$, a process $X \in \mathscr{D}_{\sigma}$ does not necessarily allow a decomposition $X=M+A$ with $M \in \mathscr{M}_{\sigma}$ and a predictable process $A \in \mathscr{V}$. Such processes are called almost special semimartingales (semimartingales presque spéciales) by Emery [6].

4. Canonical representation of $\sigma$-martingales. Local martingales $X$ can be uniquely written as a sum of a continuous and a purely discontinuous local martingales. More precisely, we have the canonical representation $X=X_{0}+X^{c}+x *(\mu-\nu)$. The goal of this section is to derive an analogous statement for $\sigma$-martingales. To this end, we generalize the definition of stochastic integrals relative to compensated random measures (cf. Definition 4.3 below).

Let $\mu$ be an integer-valued random measure on $\left(\mathbf{R}_{+} \times E, \mathscr{B}_{+} \otimes \mathscr{E}\right)$ with compensator $\nu$. Moreover, let $A \in \mathscr{A}_{\text {loc }}^{+}$be a predictable process and $F$ a transition kernel from $\left(\Omega \times \mathbf{R}_{+}, \mathscr{P}\right)$ into $(E, \mathscr{E})$ with $\nu=A \otimes F$ (cf. [4, II.1.8]). Recall that we use the notation of [4], in particular Chapter II.

Definition 4.1. We denote by $L_{\sigma}(\nu)$ the set of all $\widetilde{\mathscr{P}}$-measurable functions $W$ on $\widetilde{\Omega}$ such that $\int|W(t, x)| F_{t}(d x)<\infty \quad(P \otimes A)$-almost everywhere and $\left(\int W(t, x) F_{t}(d x)\right)_{t \in \mathbf{R}_{+}} \in L(A)$. For $W \in L_{\sigma}(\nu)$ we define $W * \nu:=$ $\int_{0} \int W(t, x) F_{t}(d x) d A_{t}$.

Alternatively, one could define this stochastic integral by $\sigma$-localization:

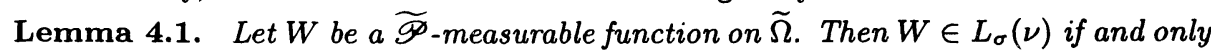
if there exist a semimartingale $X$ and an increasing sequence $\left(D_{n}\right)_{n \in \mathbb{N}}$ of predictable sets such that $D_{n} \uparrow \Omega \times \mathbf{R}_{+}$up to an evanescent set, $\left|W 1_{D_{n}}\right| * \nu \in \mathscr{V}$, and $X^{D_{n}}=W 1_{D_{n}} * \nu$ for any $n \in \mathbf{N}$. In this case $X=W * \nu$.

$\mathrm{P}$ r o of. $\Rightarrow$ : Let $D_{n}:=\left\{(\omega, t) \in \Omega \times \mathbf{R}_{+}:\left(\int|W(t, x)| F_{t}(d x)\right)(\omega) \leqslant n\right\}$ for any $n \in \mathbf{N}$ and $X:=W * \nu$.

$\Leftarrow$ : By $[9,(2.74)], X$ is indistinguishable from a predictable process and hence it is a special semimartingale (e.g., by [4, I.4.23 and I.3.10]). If we denote its local martingale part by $M$, we have $M^{D_{n}}=0$ for any $n \in \mathbf{N}$ and hence $M=0$ up to indistinguishability (again by $[9,(2.74)]$ ). Therefore $X \in \mathscr{V}$. For its variation process, we have

$$
\begin{aligned}
1_{D_{n}} \cdot \operatorname{Var}(X) & =\operatorname{Var}\left(X^{D_{n}}\right)=\operatorname{Var}\left(W 1_{D_{n}} * \nu\right)=\operatorname{Var}\left(\int_{0}^{\cdot} 1_{D_{n}}(t) \int W(t, x) F_{t}(d x) d A_{t}\right) \\
& =\int_{0} 1_{D_{n}}(t)\left|\int W(t, x) F_{t}(d x)\right| d A_{t}
\end{aligned}
$$

for any $n \in \mathbf{N}$, which implies that $\int_{0}\left|\int W(t, x) F_{t}(d x)\right| d A_{t}=\operatorname{Var}(X) \in \mathscr{V}$ by monotone convergence. In particular, $\int W(t, x) F_{t}(d x) \in L(A)$. The equality $X=W * \nu$ follows from the first part of the proof.

Lemma 4.1 is proved. 
$\mathrm{D}$ e $\mathrm{f}$ i $\mathrm{n}$ it i o n 4.2. A $\sigma$-martingale $M$ is called purely discontinuous if $M_{0}=0$ and $M N \in \mathscr{M}_{\sigma}$ for any continuous $\sigma$-martingale $N$.

Proposition 4.1. 1. A local martingale is purely discontinuous in the sense of Definition 4.2 if and only if it is purely discontinuous in the sense of [4, I.4.11].

2. $M \in \mathscr{M}_{\sigma}$ is purely discontinuous if and only if $M_{0}=0$ and $[M, N] \in \mathscr{M}_{\sigma}$ for any continuous $N \in \mathscr{M}_{\sigma}$.

3. $M$ is a purely discontinuous $\sigma$-martingale if and only if it belongs $\sigma$-locally to the class of purely discontinuous local martingales.

4. Suppose that $M \in \mathscr{V}$ can be written as $M=\sum_{t \leqslant .} \Delta M_{t} 1_{D}(t)$ for some predictable thin set $D$. If $E\left(\mid \Delta M_{T} \| \mathscr{F}_{T-}\right)<\infty$ and $E\left(\Delta M_{T} \mid \mathscr{F}_{T-}\right)=0$ P-almost everywhere on $\{T<\infty\}$ for any predictable stopping time $T$, then $M$ is a purely discontinuous $\sigma$ martingale.

P r o o f. $1 . \Rightarrow:$ We have to show that $M N \in \mathscr{M}_{\text {loc }}$ if $N$ is continuous, $M \in \mathscr{M}_{\text {loc }}$, $M_{0}=0, M N \in \mathscr{M}_{\sigma}$. In view of Corollary 3.1, it suffices to prove that $M N$ is a special semimartingale. Define stopping times $T_{n}:=\inf \left\{t \in \mathbf{R}_{+}:\left|N_{t}\right|>n\right\}$ for any $n \in \mathbf{N}$. Since $\sup _{t \leqslant} \cdot\left|(M N)_{t}^{T_{n}}\right| \leqslant n \sup _{t \leqslant} \cdot\left|M_{t}^{T_{n}}\right| \in \mathscr{A}_{\text {loc }}^{+}$, it follows that $(M N)^{T_{n}}$ is special (cf. $[4$, I.4.23]). Since locally special semimartingales are special (cf. [4, I.4.25]), we are done.

$\Leftarrow$ : Note that $N-N_{0} \in \mathscr{M}_{\text {loc }}$ for any continuous $\sigma$-martingale $N$ (cf. Corollary 3.2). Moreover $M N_{0} \in \mathscr{M}_{\sigma}$ for any $M \in \mathscr{M}_{\sigma}$ because $N_{0}$ is $\sigma$-locally bounded.

2. This follows immediately from $M N=M_{0} N_{0}+M_{-} \cdot N+N_{-} \cdot M+[M, N]$ and Lemma 3.3.

3. Since $[M, N]^{D}=1_{D} \cdot[M, N]=\left[1_{D} \cdot M, N\right]=\left[M^{D}, N\right]$ for any predictable set $D$, this follows easily from Statement 2 .

4. Let $\left(T_{n}\right)_{n \in \mathbf{N}}$ be a sequence of predictable stopping times with $D=\bigcup_{n \in \mathbf{N}} \llbracket T_{n} \rrbracket$ up to an evanescent set (cf. $[4, \mathrm{I} .2 .23])$. For any $n \in \mathbf{N}$ define a predictable set $D_{n}:=$ $D^{C} \cup\left(\bigcup_{m \leqslant n} \llbracket T(m, n) \rrbracket\right)$, where

$$
T(m, n)(\omega):= \begin{cases}T_{m}(\omega) & \text { if } E\left(\left|\Delta M_{T_{m}}\right| \mid \mathscr{F}_{T_{m}-}\right)(\omega) \leqslant n, \\ \infty & \text { otherwise. }\end{cases}
$$

$\left(D_{n}\right.$ is predictable by $\left[4, \quad\right.$ I.2.10].) Since $M^{D_{n}}=\sum_{m \leqslant n} \Delta M_{T_{m}} 1_{\llbracket T(m, n), \infty \mathbb{I}}$, we have that $M^{D_{n}}$ is a purely discontinuous local martingale by $[9,(1.45)]$ and $[4$, I.4.14]. In view of Statement 3, we are done.

$\mathrm{D}$ e f i $\mathrm{n}$ i $\mathrm{t}$ i o $\mathrm{n} 4.3$. Let $W$ be a $\widetilde{\mathscr{P}}$-measurable function on $\widetilde{\Omega}$. We say that $W \in G_{\sigma}(\mu)$ if there exists a purely discontinuous $\sigma$-martingale $M$ such that $\Delta M$ and $\widetilde{W}$ are indistinguishable. In this case, we set $W *(\mu-\nu):=M$.

Lemma 4.2. Let $W \in G_{\sigma}(\mu)$. Then we have:

1. The stochastic integral in the previous definition is well defined;

2. $W \in G_{\text {loc }}(\mu)$ if and only if $W *(\mu-\nu) \in \mathscr{M}_{\text {loc }}$.

P r o of. 1 . Let $\bar{M}$ be a purely discontinuous $\sigma$-martingale with the same jumps as $M$. Then $M-\bar{M}$ is a continuous $\sigma$-martingale and hence a local martingale which is both continuous and purely discontinuous (cf. Corollary 3.2). By [4, I.4.13], it follows that $M=\bar{M}$ up to indistinguishability.

2. $\Rightarrow$ : This is obvious.

$\Leftarrow$ : By $[4$, I.4.52 and I.4.55] we have that

$$
\sqrt{\sum_{t \leqslant}\left(\widetilde{\left.W_{t}\right)^{2}}\right.}=\sqrt{\sum_{t \leqslant}\left(\Delta(W *(\mu-\nu))_{t}\right)^{2}} \leqslant \sqrt{[W *(\mu-\nu), W *(\mu-\nu)]} \in \mathscr{A}_{\mathrm{loc}}^{+},
$$

which yields the claim.

Lemma 4.2 is proved.

The following result corresponds to Lemmas 2.2 and 4.1 .

Lemma 4.3. Let $W$ be a $\widetilde{\mathscr{P}}$-measurable function on $\widetilde{\Omega}$. Then $W \in G_{\sigma}(\mu)$ if and only if there exist a semimartingale $X$ and an increasing sequence $\left(D_{n}\right)_{n \in \mathbf{N}}$ of predictable sets such that $D_{n} \uparrow \Omega \times \mathbf{R}_{+}$up to an evanescent set, $W 1_{D_{n}} \in G_{\text {loc }}(\mu)$, and $X^{D_{n}}=$ $W 1_{D_{n}} *(\mu-\nu)$ for any $n \in \mathbf{N}$. In this case $X=W *(\mu-\nu)$. 
P r o o f. $\Rightarrow$ : Let $\left(D_{n}\right)_{n \in \mathbf{N}}$ be a $\sigma$-localizing sequence of $W *(\mu-\nu)$ in the sense of Proposition 4.1, Statement 3. Then $(W *(\mu-\nu))^{D_{n}}$ is a purely discontinuous local martingale with jumps $\widetilde{W} 1_{D_{n}}=\left(W 1_{D_{n}}\right)^{\sim}$, which implies that $W 1_{D_{n}} \in G_{\text {loc }}(\mu)$ and $(W *(\mu-\nu))^{D_{n}}=W 1_{D_{n}} *(\mu-\nu)($ cf. Lemma 4.2).

$\Leftarrow$ : Obviously, $X$ has jumps $\Delta X=\left(W 1_{D_{n}}\right)^{\sim}=\widetilde{W} 1_{D_{n}}=\widetilde{W}$ on any $D_{n}$, which implies that $\Delta X=\widetilde{W}$ up to indistinguishability. By Statement 3 of Proposition 4.1, $X$ is a purely discontinuous $\sigma$-martingale.

Lemma 4.3 is proved.

Definition 4.3 and Lemma 4.3 do not provide an explicit condition for the integrability of a predictable function $W$. Such a condition should ideally be expressed only in terms of the compensator $\nu$ of $\mu$. It is provided by the following lemma.

Lemma 4.4. Let $W$ be a $\widetilde{\mathscr{P}}$-measurable function on $\widetilde{\Omega}$. Let

$$
\begin{aligned}
W^{\prime} & :=(W-\widehat{W}) 1_{\{|W-\widehat{W}| \leqslant 1\}}+\widehat{W} 1_{\{|\widehat{W}| \leqslant 1\}}, \\
W^{\prime \prime} & :=(W-\widehat{W}) 1_{\{|W-\widehat{W}|>1\}}, \\
W^{\prime \prime \prime} & :=W^{\prime \prime}-(1-a) W 1_{\{|\widehat{W}|>1\}} .
\end{aligned}
$$

Then we have equivalence between:

1. $W \in G_{\sigma}(\mu)$

2. (a) $\widehat{W}$ is finite outside some evanescent set,

(b) $C\left(W^{\prime}\right) \in \mathscr{V}$, where $C\left(W^{\prime}\right):=\left(W^{\prime}-\widehat{W}^{\prime}\right)^{2} * \nu+\sum_{t \leqslant \cdot} \cdot\left(1-a_{t}\right)\left(\widehat{W}_{t}^{\prime}\right)^{2}$,

(c) $1_{\left\{W^{\prime \prime}(x) \neq 0\right\}} * \nu \in \mathscr{V}$,

(d) $\sum_{t \leqslant .} \cdot\left(1-a_{t}\right) 1_{\left\{\left|\widehat{W}_{t}\right|>1\right\}} \in \mathscr{V}$

(e) $W^{\prime \prime \prime} \in L_{\sigma}(\nu)$.

In this case

$$
W *(\mu-\nu)=W^{\prime} *(\mu-\nu)+W^{\prime \prime} * \mu-\sum_{t \leqslant \cdot}(1-\mu(\{t\} \times E)) \widehat{W}_{t} 1_{\left\{\left|\widehat{W}_{t}\right|>1\right\}}-W^{\prime \prime \prime} * \nu .
$$

$\mathrm{P}$ r o o f. $1 \Rightarrow 2$ : Let $X:=W *(\mu-\nu)$. By [4, II.2.34], the canonical semimartingale representation of $X$ is

$$
X=X^{c}+h *\left(\mu^{X}-\nu^{X}\right)+\bar{h} * \mu^{X}+B
$$

where $\mu^{X}, \nu^{X}$ denote the measure of jumps of $X$ and its compensator, $B \in \mathscr{V}$ is some predictable process, and $h(x):=x 1_{\{|x| \leqslant 1\}}, \bar{h}(x):=x-h(x)$ for $x \in \mathbf{R}$.

Let $\left(D_{n}\right)_{n \in \mathrm{N}}$ be a $\sigma$-localizing sequence for $X$ as in Lemma 4.3. Fix $n \in \mathbf{N}$. From $W 1_{D_{n}} \in G_{\text {loc }}(\mu)$ it follows that $\widehat{W}$ is finite on $D_{n}$.

Since $\Delta X=\widetilde{W}$ and by definition of $\widetilde{W}$ in [4, II.1.27], we have

$$
\begin{aligned}
\bar{h} 1_{D_{n}} * \mu^{X} & =\sum_{t \leqslant} \bar{h}\left(\Delta X_{t}\right) 1_{D_{n}}(t)=\sum_{t \leqslant \cdot} \bar{h}\left(\widetilde{W}_{t}\right) 1_{D_{n}}(t) \\
& =\bar{h}(W-\widehat{W}) 1_{D_{n}} * \mu+\sum_{t \leqslant \cdot}(1-\mu(\{t\} \times E)) \bar{h}\left(-\widehat{W}_{t}\right) 1_{D_{n}}(t) \\
& =W^{\prime \prime} 1_{D_{n}} * \mu-\sum_{t \leqslant}(1-\mu(\{t\} \times E)) \widehat{W}_{t} 1_{\left\{\left|\widehat{W}_{t}\right|>1\right\}} 1_{D_{n}}(t) .
\end{aligned}
$$

From $X^{D_{n}}=\left(X^{c}\right)^{D_{n}}+h 1_{D_{n}} *\left(\mu^{X}-\nu^{X}\right)+\bar{h} 1_{D_{n}} * \mu^{X}+B^{D_{n}} \in \mathscr{M}_{\text {loc }}$ it follows that $\bar{h} 1_{D_{n}} * \mu^{X}+B^{D_{n}} \in \mathscr{M}_{\text {loc }} \cap \mathscr{A}_{\text {loc }}$ and hence $\bar{h} 1_{D_{n}} * \mu^{X} \in \mathscr{A}_{\text {loc }}$ by [4, I.4.23 and I.3.10]. By [4, II.1.8], this implies $-B^{D_{n}}=\bar{h} 1_{D_{n}} * \nu^{X}$. This process in turn equals $W^{\prime \prime} 1_{D_{n}} *$ $\nu-\sum_{t \leqslant} \cdot\left(1-a_{t}\right) \widehat{W}_{t} 1_{\left\{\left|\widehat{W}_{t}\right|>1\right\}} 1_{D_{n}}(t)=\left(W^{\prime \prime}-(1-a) W 1_{\{|\widehat{W}|>1\}}\right) 1_{D_{n}} * \nu=W^{\prime \prime \prime} 1_{D_{n}} * \nu$ by equation (4.2) and $[9,(3.67)]$. It follows that $W^{\prime \prime \prime} \in L_{\sigma}(\nu)$ and $B=-W^{\prime \prime \prime} * \nu$ by Lemma 4.1.

Similarly as equation (4.2), it follows that $1_{\{\bar{h}(x) \neq 0\}} * \mu^{X}=1_{\left\{W^{\prime \prime}(x) \neq 0\right\}} * \mu+\sum_{t \leqslant} .(1-$ $\mu(\{t\} \times E)) 1_{\left\{\left|\widehat{W}_{t}\right|>1\right\}}$. This process counts the «big» jumps of $X$. Since $X$ has only 
finitely many big jumps on any finite interval, it follows that the process $1_{\{\bar{h}(x) \neq 0\}} * \mu^{X}$ and hence both terms on the right-hand side are in $\mathscr{A}_{\text {loc }}$. By $[9,(3.67)]$, this implies $1_{\left\{W^{\prime \prime}(x) \neq 0\right\}} * \nu \in \mathscr{V}$ and $\sum_{t \leqslant .}\left(1-a_{t}\right) 1_{\left\{\left|\widehat{W}_{t}\right|>1\right\}} \in \mathscr{V}$.

Similarly as equation (4.2), it follows that

$$
h 1_{D} * \mu^{X}=h(W-\widehat{W}) 1_{D} * \mu+\sum_{t \leqslant \cdot}(1-\mu(\{t\} \times E)) h\left(-\widehat{W}_{t}\right) 1_{D}(t)
$$

for any predictable set $D$ with $|h| 1_{D} * \mu^{X} \in \mathscr{V}$. Fix $t \in \mathbf{R}_{+}$. Since $\int|h| \nu^{X}(\{t\} \times d x) \leqslant 1$, we have that $\left|h 1_{D}\right| * \nu^{X} \in \mathscr{A}_{\text {loc }}^{+}$and hence $\left|h 1_{D}\right| * \mu^{X} \in \mathscr{A}_{\text {loc }}^{+}$for $D:=\Omega \times\{t\}$. In view of $[9,(3.67)]$, calculating the compensator at time $t$ of both sides of equation (4.3) yields

$$
\begin{aligned}
\widehat{h}_{t} & :=\int h(x) \nu^{X}(\{t\} \times d x)=\int h\left(W(t, x)-\widehat{W}_{t}\right) \nu(\{t\} \times d x)+\left(1-a_{t}\right) h\left(-\widehat{W}_{t}\right) \\
& =\int\left(h\left(W(t, x)-\widehat{W}_{t}\right)-h\left(-\widehat{W}_{t}\right)\right) \nu(\{t\} \times d x)+h\left(-\widehat{W}_{t}\right)=\widehat{W}_{t}^{\prime}+h\left(-\widehat{W}_{t}\right) .
\end{aligned}
$$

Similarly as equation (4.2), it follows that

$$
\begin{aligned}
& (h-\widehat{h})^{2} * \mu^{X}+\sum_{t \leqslant \cdot}\left(1-\mu^{X}(\{t\} \times \mathbf{R})\right) \widehat{h}_{t}^{2}=\sum_{t \leqslant \cdot}\left(h\left(\Delta X_{t}\right)-\widehat{h}_{t}\right)^{2} \\
& =(h(W-\widehat{W})-\widehat{h})^{2} * \mu+\sum_{t \leqslant \cdot}(1-\mu(\{t\} \times E))\left(h\left(-\widehat{W}_{t}\right)-\widehat{h}_{t}\right)^{2} \\
& =\left(W^{\prime}-\widehat{W}^{\prime}\right)^{2} * \mu+\sum_{t \leqslant}(1-\mu(\{t\} \times E))\left(-W_{t}^{\prime}\right)^{2} .
\end{aligned}
$$

Since $h *\left(\mu^{X}-\nu^{X}\right)$ has bounded jumps, it is in $\mathscr{H}_{\text {loc }}^{2}$. By [4, II.1.33], this implies that $(h-\widehat{h})^{2} * \nu^{X}+\sum_{t \leqslant} \cdot\left(1-\nu^{X}(\{t\} \times \mathbf{R})\right) \widehat{h}_{t}^{2} \in \mathscr{A}_{\text {loc }}^{+}$. By [9, (3.67)], this process is the compensator of the left-hand side of equation (4.4). Also by [9, (3.67)], we obtain $\left(W^{\prime}-\widehat{W}^{\prime}\right)^{2} * \nu+$ $\sum_{t \leqslant .} \cdot\left(1-a_{t}\right)\left(\widehat{W}_{t}^{\prime}\right)^{2} \in \mathscr{A}_{\text {loc }}^{+}$for the compensator of the right-hand side of equation (4.4).

$2 \Rightarrow 1$ : By $\left[4\right.$, II.1.33] and $[9,(3.67)], X:=W^{\prime} *(\mu-\nu)+W^{\prime \prime} * \mu-W^{\prime \prime \prime} * \nu-\sum_{t \leqslant} .(1-$ $\mu(\{t\} \times E)) \widehat{W}_{t} 1_{\left\{\left|\widehat{W}_{t}\right|>1\right\}}$ is a well-defined semimartingale. Let $\left(D_{n}\right)_{n \in \mathbf{N}}$ be a $\sigma$-localizing sequence for $W^{\prime \prime \prime} \in L_{\sigma}(\nu)$ in the sense of Lemma 4.1. Fix $n \in \mathbf{N}$. By [4, II.1.28], we have that $W^{\prime \prime \prime} 1_{D_{n}} \in G_{\mathrm{loc}}(\mu)$ and $W^{\prime \prime \prime} 1_{D_{n}} * \mu-W^{\prime \prime \prime} 1_{D_{n}} * \nu=W^{\prime \prime \prime} 1_{D_{n}} *(\mu-\nu)$. Observe that

$$
\begin{aligned}
& X^{D_{n}}-\left(W^{\prime}+W^{\prime \prime \prime}\right) 1_{D_{n}} *(\mu-\nu) \\
& \quad=\sum_{t \leqslant}\left(\left(1-a_{t}\right) \int W(t, x) \mu(\{t\} \times d x)-(1-\mu(\{t\} \times E)) \widehat{W}_{t}\right) 1_{\left\{\left|\widehat{W}_{t}\right|>1\right\}} 1_{D_{n}}(t) .
\end{aligned}
$$

If we denote this process by $M$, then we have $E\left(\Delta M_{T} \mid \mathscr{F}_{T-}\right)=\left(\left(1-a_{T}\right) \widehat{W}_{T}-\right.$ $\left.\left(1-a_{T}\right) \widehat{W}_{T}\right) 1_{\left\{\left|\widehat{W}_{T}\right|>1\right\}} 1_{D_{n}}(T)=0$ on $\{T<\infty\}$ for any predictable stopping time $T$ (cf. [4, II.1.26]). From Proposition 4.1 it follows that $M$ and hence $X^{D_{n}}$ is a purely discontinuous $\sigma$-martingale. By Propositions 4.1 and 2.1 applied to the class of purely discontinuous local martingales, this is also true for $X$. For the jumps we have

$$
\begin{aligned}
\Delta X_{t}= & \left(W^{\prime}+W^{\prime \prime \prime}\right)_{t}^{\sim}+\left(1-a_{t}\right) \int W(t, x) \mu(\{t\} \times d x) 1_{\left\{\left|\widehat{W}_{t}\right|>1\right\}} \\
& -(1-\mu(\{t\} \times E)) \widehat{W}_{t} 1_{\left\{\left|\widehat{W}_{t}\right|>1\right\}} \\
= & \left(W^{\prime}+W^{\prime \prime \prime}\right)_{t} \tilde{t}+\left(1-a_{t}\right) \widetilde{W}_{t} 1_{\left\{\left|\widehat{W}_{t}\right|>1\right\}}+\widetilde{1}_{t} \widehat{W}_{t} 1_{\left\{\left|\widehat{W}_{t}\right|>1\right\}} \\
= & \left(W^{\prime}+W^{\prime \prime \prime}+(1-a) W 1_{\{|\widehat{W}|>1\}}+\widehat{W} 1_{\{|\widehat{W}|>1\}}\right)_{t}=\widetilde{W}_{t}
\end{aligned}
$$

on $D_{n}$ and hence outside some evanescent set. It follows that $W \in G_{\sigma}(\mu)$ and $X=$ $W *(\mu-\nu)$.

Lemma 4.4 is proved.

The following lemma extends [4, II.1.30] and [7, Proposition A.1.5] to general stochastic integration. 
Lemma 4.5. Let $W=\left(W^{1}, \ldots, W^{d}\right)$, where $W^{i} \in G_{\sigma}(\mu)$ for $i=1, \ldots, d$. Moreover let $H$ be an $\mathbf{R}^{d}$-valued predictable process. Then $H^{\top} W \in G_{\sigma}(\mu)$ if and only if $H \in$ $L(W *(\mu-\nu))$. In this case $\left(H^{\top} W\right) *(\mu-\nu)=H^{\top} \cdot(W *(\mu-\nu))$, where the stochastic integral of $W$ is to be understood componentwise.

P r o o f. $\Rightarrow$ : Define $X:=\left(H^{\top} W\right) *(\mu-\nu)$ and let $\left(D_{n}\right)_{n \in \mathbf{N}},\left(D_{n}^{\prime}\right)_{n \in \mathbf{N}}$ be $\sigma$-localizing sequences as in Lemma 4.3 for $H^{\top} W \in G_{\sigma}(\mu)$ and $W \in G_{\sigma}(\mu)$, respectively. Without loss of generality $H$ is bounded on $D_{n}$ for any $n \in \mathbf{N}$. Then we have

$$
\begin{aligned}
X^{D_{n} \cap D_{n}^{\prime}} & =1_{D_{n}^{\prime}} \cdot\left(\left(H^{\top} W 1_{D_{n}}\right) *(\mu-\nu)\right) \\
& =\left(H^{\top} W 1_{D_{n} \cap D_{n}^{\prime}}\right) *(\mu-\nu) \\
& =\left(H 1_{D_{n}}\right)^{\top} \cdot\left(\left(W 1_{D_{n}^{\prime}}\right) *(\mu-\nu)\right) \\
& =\left(H 1_{D_{n}}\right)^{\top} \cdot\left(1_{D_{n}^{\prime}} \cdot(W *(\mu-\nu))\right) \\
& =\left(H 1_{D_{n} \cap D_{n}^{\prime}}\right)^{\top} \cdot(W *(\mu-\nu))
\end{aligned}
$$

by [7, Proposition A.1]. In view of Lemma 2.2, the claim follows.

$\Leftarrow$ : Define $X:=H^{\top} \cdot(W *(\mu-\nu))$. Let $\left(D_{n}\right)_{n \in \mathbf{N}},\left(D_{n}^{\prime}\right)_{n \in \mathbf{N}}$ be $\sigma$-localizing sequences as in Lemma 2.2 for $H \in L(W *(\mu-\nu))$ and as in Lemma 4.3 for $W \in G_{\sigma}(\mu)$, respectively. Then we have

$$
\begin{aligned}
X^{D_{n} \cap D_{n}^{\prime}} & =1_{D_{n}^{\prime}} \cdot\left(\left(H 1_{D_{n}}\right)^{\top} \cdot(W *(\mu-\nu))\right) \\
& =\left(H 1_{D_{n} \cap D_{n}^{\prime}}\right)^{\top} \cdot(W *(\mu-\nu)) \\
& =\left(H 1_{D_{n}}\right)^{\top} \cdot\left(1_{D_{n}^{\prime}} \cdot(W *(\mu-\nu))\right) \\
& =\left(H 1_{D_{n}}\right)^{\top} \cdot\left(\left(W 1_{D_{n}^{\prime}}\right) *(\mu-\nu)\right) \\
& =\left(H^{\top} W 1_{D_{n} \cap D_{n}^{\prime}}\right) *(\mu-\nu)
\end{aligned}
$$

by [7, Proposition A.1]. In view of Lemma 4.3, the claim follows.

Lemma 4.5 is proved.

Finally, we state and prove the main result of this section.

Theorem 4.1. Any $\mathbf{R}^{d}$-valued $\sigma$-martingale $X$ can be written as

$$
X=X_{0}+X^{c}+x *(\mu-\nu),
$$

where $\mu$ denotes the measure of jumps of $X$ and the stochastic integral of $x=\left(x^{1}, \ldots, x^{d}\right)$ is to be understood componentwise.

$\mathrm{P} \mathrm{r}$ o o f. Without loss of generality $d=1$. The canonical semimartingale representation of $X$ is $X=X_{0}+X^{c}+h *(\mu-\nu)+(x-h(x)) * \mu+B$ for some predictable process $B \in \mathscr{V}$ and some truncation function $h: \mathbf{R}^{d} \rightarrow \mathbf{R}^{d}$ (cf. [4, II.2.34]). Let $\left(D_{n}\right)_{n \in \mathbf{N}}$ denote a $\sigma$-localizing sequence of $X$. Fix $n \in \mathrm{N}$. Since $X^{D_{n}}=X_{0}^{D_{n}}+\left(X^{c}\right)^{D_{n}}+h 1_{D_{n}} *(\mu-\nu)+(x-$ $h(x)) 1_{D_{n}} * \mu+B^{D_{n}}$ is a local martingale, we have that $(x-h(x)) 1_{D_{n}} * \mu \in \mathscr{A}_{\text {loc }}$ and its compensator $(x-h(x)) 1_{D_{n}} * \nu \in \mathscr{A}_{\text {loc }}$ coincides with $-B^{D_{n}}$ (cf. [4, I.4.23]). By [4, II.1.28], $(x-h(x)) 1_{D_{n}} \in G_{\mathrm{loc}}(\mu)$ and $(x-h(x)) 1_{D_{n}} * \mu+B^{D_{n}}=(x-h(x)) 1_{D_{n}} *(\mu-\nu)$, which implies that $\left(X-X_{0}-X^{c}\right)^{D_{n}}=x 1_{D_{n}} *(\mu-\nu)$. In view of Lemma 4.3, it follows that $x \in G_{\sigma}(\mu)$ and $x *(\mu-\nu)=X-X_{0}-X^{c}$. Theorem 4.1 is proved.

5. Equivalent $\sigma$-martingale measures. Delbaen and Schachermayer [5] relate the absence of free lunches to the existence of equivalent $\sigma$-martingale measures. Below we show how these measures can be characterized in terms of semimartingale characteristics. To this end, let $S=\left(S^{1}, \ldots, S^{d}\right)$ be an $\mathbf{R}^{d}$-valued semimartingale which stands for the discounted prices of securities $1, \ldots, d$. Moreover, let $P^{\star} \stackrel{\text { loc }}{\sim} P$ be a probability measure with density process $Z$. By $N:=\mathscr{L}(Z):=\left(1 / Z_{-}\right) \cdot Z$ we denote the stochastic logarithm of $Z$, i.e., the unique semimartingale $N$ with $\mathscr{E}(N)=Z$ (cf. [13] for details on the 
stochastic logarithm; note that $Z$ and $Z_{-}$are positive by $P^{\star} \stackrel{\text { loc }}{\sim} P$ and $\left.[4,1.2 .27]\right)$. Suppose that the characteristics $(B, C, \nu)$ of the $\mathbf{R}^{d+1}$-valued semimartingale $(S, N)$ relative to $h: \mathbf{R}^{d+1} \rightarrow \mathbf{R}^{d+1}$ are given in the form

$$
B=b \cdot A, \quad C=c \cdot A, \quad \nu=A \otimes F,
$$

where $A \in \mathscr{A}_{\text {loc }}^{+}$is a predictable process, $b$ is a predictable $\mathbf{R}^{d+1}$-valued process, $c$ is a predictable $\mathbf{R}^{(d+1) \times(d+1)}$-valued process whose values are nonnegative, symmetric matrices, and $F$ is a transition kernel from $\left(\Omega \times \mathbf{R}_{+}, \mathscr{P}\right)$ into $\left(\mathbf{R}^{d+1}, \mathscr{B}^{d+1}\right)$.

The Girsanov-Jacod-Mémin theorem as stated in [4, III.3.24] indicates how the characteristics change if $P$ is replaced with $P^{\star}$. Here, we formulate this result in terms of the joint characteristics of $(S, N)$, which is convenient for applications.

Lemma 5.1. The $P^{\star}$-characteristics of $(S, N)$ are of the form (5.1), but with

$$
b^{\star}=b+c^{\cdot, d+1}+\int h(x) x^{d+1} F(d x), \quad c^{\star}=c, \quad \frac{d F^{\star}}{d F}(x)=1+x^{d+1}
$$

instead of $b, c, F$.

$\mathrm{P}$ r o o f. According to [4, III.3.31], there exists a predictable $\mathbf{R}^{d+1}$-valued process $\beta$ such that $\beta^{\top} c \beta \in L(A)$ and $c^{i, d+1} \cdot A=\left\langle S^{i, c}, N^{c}\right\rangle=\left(c^{i \cdot} \beta\right) \cdot A$ for $i=1, \ldots, d$. It follows that $\left\langle Z^{c}, S^{i, c}\right\rangle=Z_{-} \cdot\left\langle N^{c}, S^{i, c}\right\rangle=\left(Z_{-} c^{i \cdot} \beta\right) \cdot A$.

Secondly, we have $Z_{t}=Z_{t-}\left(1+\Delta N_{t}\right)=Z_{t-}\left(1+x^{d+1}\right)$ for $\mu^{(S, N)}$-almost all $(t, x) \in \mathbf{R}_{+} \times \mathbf{R}^{d+1}$, which implies that $E\left(Z U * \mu_{\infty}^{(S, N)}\right)=E\left(\left(1+x^{d+1}\right) Z_{-} U * \mu_{\infty}^{(S, N)}\right)$ for any nonnegative $\left(\mathscr{P} \otimes \mathscr{B}^{d+1}\right)$-measurable function $U$. Put differently, we have $Y Z_{-}=M_{\mu}^{P(s, N)}(Z \mid \widetilde{\mathscr{P}})$ in the sense of $\left[4\right.$, III.3c] for $Y(\omega, t, x):=1+x^{d+1}$. By [4, III.3.24], we are done.

$\mathrm{D}$ e f i n it i o $\mathrm{n}$ 5.1. We call $P^{\star}$ an equivalent martingale measure (EMM) (respectively, equivalent local martingale measure (ELMM), equivalent $\sigma$-martingale measure $(E \sigma M M))$ if $S^{i}$ is a martingale (respectively, local martingale, $\sigma$-martingale) relative to $P^{\star}$ for $i=1, \ldots, d$.

$\mathrm{R}$ e $\mathrm{m}$ a $\mathrm{rk}$. In the paper by Delbaen and Schachermayer [4] the term equivalent local martingale measure is used in the above sense. Kramkov and Schachermayer [15] and Becherer [1], however, apply the same name to denote measures $P^{\star} \sim P$ such that $1+\varphi^{\top} \cdot S$ is a $P^{\star}$-local martingale for any $\varphi \in L(S)$ with $1+\varphi^{\top} \cdot S \geqslant 0$. In order to avoid confusion, we prefer to call measures of such kind equivalent weak local martingale measures. One easily shows that any $\sigma$-martingale measure is a weak local martingale measure while the converse is not true. Nevertheless, the existence of an equivalent weak local martingale measure suffices to ensure condition NFLVR (cf. [1, Proposition 2.3]).

For the proof of Lemma 5.2 we need the following proposition, which extends [4, III.3.8] to the $\sigma$-martingale case.

Proposition 5.1. A real-valued semimartingale $X$ is a $P^{\star}$-martingale (respectively, $P^{\star}$-local martingale, $P^{\star}-\sigma$-martingale) if and only if $X Z$ is a $P$-martingale (respectively, $P$-local martingale, $P$ - $\sigma$-martingale).

$\mathrm{P}$ r o o f. By [4, III.3.8], it suffices to prove the assertion for $\sigma$-martingales. Let $\left(D_{n}\right)_{n \in \mathbf{N}}$ be an increasing sequence of predictable sets. By partial integration we have

$$
\begin{aligned}
1_{D_{n}} \cdot(X Z) & =1_{D_{n}} \cdot\left(X_{-} \cdot Z\right)+1_{D_{n}} \cdot\left(Z_{-} \cdot X\right)+1_{D_{n}} \cdot[X, Z] \\
& =\left(1_{D_{n}} X_{-}\right) \cdot Z+\left(1_{D_{n}} \cdot X\right) Z-\left(1_{D_{n}} \cdot X\right)_{-} \cdot Z .
\end{aligned}
$$

$\Rightarrow$ : Let $\left(D_{n}\right)_{n \in \mathbf{N}}$ be a $\sigma$-localizing sequence for the $P^{*}-\sigma$-martingale $X$. Then $1_{D_{n}}$. $(X Z)$ is a $P$-local martingale. Therefore $X Z \in\left(\mathscr{M}_{\text {loc }}^{P}\right)_{\sigma}=\mathscr{M}_{\sigma}^{P}$, i.e., it is a $P$ - $\sigma$-martingale.

$\Leftarrow$ : This follows similarly if $\left(D_{n}\right)_{n \in \mathbf{N}}$ now denotes a $\sigma$-localizing sequence for the $P$ - $\sigma$-martingale $X Z$.

We are now ready to characterize EMM's, ELMM's, and E $\sigma$ MM's in terms of semimartingale characteristics: 
Lemma 5.2. 1. $S$ is a $P^{\star}$-martingale if and only if $S Z \in \mathscr{D}$ relative to $P$ and

$$
b^{i}+c^{i, d+1}+\int\left(x^{i}\left(1+x^{d+1}\right)-h^{i}(x)\right) F(d x)=0 \quad \text { for } \quad i=1, \ldots, d
$$

holds $(P \otimes A)$-almost everywhere on $\Omega \times \mathbf{R}_{+}$.

2. $S$ is a $P^{\star}$-local martingale if and only if $\int_{\left\{\left|x^{i}\right|>1\right\}}\left|x^{i}\left(1+x^{d+1}\right)\right| F(d x) \in L(A)$ for $i=1, \ldots, d$ and condition (5.2) holds $(P \otimes A)$-almost everywhere on $\Omega \times \mathbf{R}_{+}$.

3. $S$ is a $P^{\star}-\sigma$-martingale if and only if $\int_{\left\{\left|x^{i}\right|>1\right\}}\left|x^{i}\left(1+x^{d+1}\right)\right| F(d x)<\infty$ for $i=$ $1, \ldots, d$ and condition (5.2) hold $(P \otimes A)$-almost everywhere on $\Omega \times \mathbf{R}_{+}$.

$\mathrm{P} \mathrm{r}$ o o f. Statements 2 and 3 follow from Lemmas 5.1 and 3.1.

1. $\Rightarrow$ : Condition (5.2) follows from Statement 2. By Proposition 5.1, $S Z$ is a $P$ martingale and hence in $\mathscr{D}$ (cf. Lemma 3.1).

$\Leftarrow$ : Fix $i \in\{1, \ldots, d\}$. Since $S^{i} Z$ and $Z$ are special semimartingales, this is also true for $Y:=\left(1 / Z_{-}\right) \cdot\left(S^{i} Z-S_{-}^{i} \cdot Z\right)=\left(1 / Z_{-}\right) \cdot\left(Z_{-} \cdot S^{i}+\left[S^{i}, Z\right]\right)=S^{i}+\left[S^{i}, N\right]$. From $\Delta Y=$ $\Delta S^{i}(1+\Delta N)$ it follows that $\left|x^{i}\left(1+x^{d+1}\right)\right| 1_{\left\{\left|x^{i}\left(1+x^{d+1}\right)\right|>1\right\}} * \nu=|x| 1_{\{|x|>1\}} * \nu^{Y} \in \mathscr{A}_{\text {loc }}$, where $\nu^{Y}$ denotes the compensator of the measure of jumps of $Y$. By [4, II.2.13], we have that $\left|x^{i}\left(1+x^{d+1}\right)\right| 1_{\left\{\left|x^{i}\right|>1\right\}} * \nu \leqslant\left|x^{i}\left(1+x^{d+1}\right)\right| 1_{\left\{\left|x^{i}\left(1+x^{d+1}\right)\right|>1\right\}} * \nu+1_{\{|x|>1\}} * \nu \in \mathscr{A}_{\text {loc }}$. Statement 2 yields that $S$ is a $P^{\star}$-local martingale, which in turn implies that $S Z$ is a $P$-local martingale. By Corollary 3.1 , we have that $S Z$ is a $P$-martingale. Once more applying Proposition 5.1 yields the claim.

Acknowledgements. Parts of this paper were originally contained in the preprint version of a joint paper with Thomas Goll, to whom I owe many fruitful discussions on the subject.

\section{REFERENCES}

1. Becherer $D$. The numeraire portfolio for unbounded semimartingales. - Finance \& Stochastics, 2001, v. 5, № 3, p. 327-341.

2. Ширяев $A . H$. , Черный $A . C$. Векторный стохастический интеграл и фундаментальные теоремы теории арбитража. - Труды матем. ин-та им. В. А. Стеклова $\mathrm{PAH}, 2002$, т. 237 , c. $12-56$.

3. Chou C. Caractérisation d'une classe de semimartingales. - Lecture Notes in Math. 1979 , v. 721 , p. $250-252$.

4. Delbaen $F$., Schachermayer $W$. A general version of the fundamental theorem of asset pricing. - Math. Ann., 1994, v. 300, № 3, p. 463-520.

5. Delbaen F., Schachermayer $W$. The fundamental theorem of asset pricing for unbounded stochastic processes. - Math. Ann., 1998, v. 312, № 2, p. 215-250.

6. Emery $M$. Compensation de processus à variation finie non localement intègrables. Lectures Notes in Math., 1980, v. 784, p. 152-160.

7. Goll T., Kallsen J. Optimal portfolios for logarithmic utility. - Stochastic Process. Appl., 2000, v. 89, № 1, p. 31-48.

8. Goll T., Kallsen J. A complete explicit solution to the log-optimal portfolio problem. - Ann. Appl. Probab. (to appear).

9. Jacod J. Calcul stochastique et problèmes de martingales. Berlin: Springer-Verlag, 1979, 539 p. (Lectures Notes in Math., v. 714.)

10. Jacod $J$. Intègrales stochastiques par rapport à une semi-martingale vectorielle et changements de filtration. - Lectures Notes in Math., 1980, v. 784, p. 161-172.

11. Жакод Ж., Ширяев А. Н. Предельные теоремы для случайных процессов. Т. 1, 2. М.: Физматлит, 1994, 544 с.; 368 с.

12. Kabanov Yu. M. On the FTAP of Kreps-Delbaen-Schachermayer. - Statistics and Control of Stochastic Processes. Ed. by Yu. M. Kabanov et al. River Edge, NJ: World Scientific, 1997, p. 191-203.

13. Kallsen J., Shiryaev A. N. The cumulant process and Esscher's change of measure. Finance \& Stochastics, 2002, v. 6, № 4, p. 397-428. 
14. Kallsen J., Shiryaev A. N. Time change representation of stochastic integrals. - Teория вероятн. и ее примен., 2001, т. 46 , в. 3 , с. 579-585.

15. Kramkov D., Schachermayer $W$. The asymptotic elasticity of utility functions and optimal investment in incomplete markets. - Ann. Appl. Probab., 1999, v. 9, № 3, p. $904-950$.

Поступила в редакцию 6.IX. 2002

(C) 2003 г.

\title{
MORDECKI E.*
}

\section{RUIN PROBABILITIES FOR LÉVY PROCESSES WITH MIXED-EXPONENTIAL NEGATIVE JUMPS ${ }^{1)}$}

\author{
To José Luis Massera (1915-2002)
}

In Memoriam

Найден явный вид вероятности разорения для процессов Леви (возможно, «убиваемых» с постоянной скоростью), отрицательные скачки которых имеют смешанное экспоненциальное распределение без ограничений на положительные скачки.

Ключевые слова и фразы: вероятность разорения, явный вид, процесс Леви, смешанное экспоненциальное распределение.

\section{Introduction.}

1.1. Let $X=\left\{X_{t}\right\}_{t \geqslant 0}$ be a real-valued stochastic process defined on a stochastic basis $\left(\Omega, \mathscr{F}, \mathbf{F}=\left(\mathscr{F}_{t}\right)_{t \geqslant 0}, \mathbf{P}\right)$ that satisfies the usual conditions. Assume that $X$ is càdlàg, adapted, $X_{0}=0$, and for $0 \leqslant s<t$ the random variable $X_{t}-X_{s}$ is independent of the $\sigma$-field $\mathscr{F}_{s}$ with a distribution that only depends on the difference $t-s$. The stochastic process $X$ is a process with stationary independent increments (PIIS) or a Lévy process. For $q \in \mathbf{R}, \psi(q)$ denotes the characteristic exponent of $X$ given by the Lévy-Khinchine formula

$$
\psi(q)=\frac{1}{t} \ln \mathbf{E} e^{i q X_{t}}=i b q-\frac{1}{2} \sigma^{2} q^{2}+\int_{\mathbf{R}}\left(e^{i q y}-1-i q y \mathbf{1}_{\{|y|<1\}}\right) \Pi(d y),
$$

where $b$ and $\sigma \geqslant 0$ are real constants and $\Pi$ is a positive measure on $\mathbf{R}-\{0\}$ such that $\int\left(1 \wedge y^{2}\right) \Pi(d y)<\infty$, called the Lévy measure. The function $\psi(q), q \in \mathbf{R}$, completely determines the law of the process. For general reference on the subject see [4], [11], or [3].

1.2. Consider now a Lévy process with measure $\Pi$ given by

$$
\Pi(d y)= \begin{cases}\pi(d y) & \text { if } \quad y>0 \\ \lambda \sum_{k=1}^{n} a_{k} \alpha_{k} e^{\alpha_{k} y} d y & \text { if } \quad y<0\end{cases}
$$

where $\pi$ is an arbitrary Lévy measure concentrated on $(0, \infty), 0<\alpha_{1}<\cdots<\alpha_{n}, a_{k}>0$, for $k=1, \ldots, n$ and $\sum_{k=1}^{n} a_{k}=1$. The magnitude of the negative jumps of $X$ is mixed exponentially distributed, with parameter $\alpha_{k}$ chosen with probability $a_{k}$, and they occur at Poissonian times with rate $\lambda$. As the process considered has a finite number of negative jumps on $[0, t]$, we consider a truncation function

$$
h(y)=y \mathbf{1}_{\{0<y<1\}} .
$$

* Facultad de Ciencias, Centro de Matemática, Iguá 4225, CP 11400, Montevideo, Uruguay; e-mail: mordecki@cmat.edu.uy

1) The research was partially supported by Project CONICYT-BID 325/95. 